50 years ago: \section{How Holonyak won the race to invent visible LEDs}

\section{Tim Palucka}

T he infinitely variable, precisely controlled riot of color that is New York's Times Square is created by a vast array of light-emitting diodes, or LEDs. Certainly this glaring display of multicolored advertising was not what Nick Holonyak Jr., then the lead scientist of General Electric's Advanced Semiconductor Laboratory in Syracuse, had in mind on October 9, 1962, when he demonstrated the first "visible" LED — a red glow from a small semiconductor crystal in an electronic circuit. But he knew his tiny device, which produced coherent red light and was thus a laser as well as an LED, would become important. In an article about lasers entitled "Light of Hope — or Terror?" in the February 1963 Reader's Digest, Harland Manchester wrote of Holonyak's invention:

The latest dramatic laser discovery, made by General Electric, may someday make the electric light bulb obsolete. While the radiation from previous lasers was invisible, this one emits visible light in the red region of the spectrum. Research is continuing, and GE engineers hope to build lasers which will convert ordinary electric current into white light with a high degree of efficiency.

"We believe there is a strong possibility of developing the laser as a practical light source," Holonyak said in the 1963 article. "Much more experimental work must be done, and it might be ten years or more before such a lamp could be ready for wide use."

Within months of the invention, General Electric was selling Holonyak's red LEDs, which produced diffuse, incoherent light - that is, light whose waves were not aligned in phase- for $\$ 260$ each. These small, glass-encapsulated cylinders, sprouting two wires that looked like legs, contained the galliumarsenide-phosphide (GaAsP) crystal that produced the light. The laser version of the same material, which emitted an intense, narrow beam of red light due to the perfect alignment, or coherence, of its waves, was arbitrarily valued at 10 times the price of the LED, or $\$ 2,600$.

Soon, red LEDs were appearing as indicators on computers and in the sevensegment displays used to form numbers in digital alarm clocks. In the 50 years since then, LEDs have been produced in a wide range of colors for use in traffic lights, architectural lighting, backlights for liquid-crystal display (LCD) television screens, and large displays, such as those in Times Square. Recent advances in high-intensity, high-efficiency white LEDs have made them useful as street lights and conventional indoor lamps. Though it took longer than the 10 years that Holonyak had predicted in 1963 , white LEDS are now poised to replace Edison's incandescent bulb, as well as fluorescent lamps.

$$
* * * * *
$$

LEDs owe their existence to two other inventions: the transistor and the ruby rod laser. The transistor, as is well known, was invented in 1947 by John Bardeen, Walter Brattain, and William Shockley at Bell Laboratories in New Jersey. The semiconductor sandwich that comprises the transistor can take a low-voltage electrical signal as an input and amplify it to produce a higher output voltage; it can also be used as an electronic switch. At the time, the vacuum tube was the established technology for these applications, which the transistor replaced due to size, cost, and reliability benefits. The invention of the transistor and its subsequent continuous miniaturization has made possible today's complex, compact electronic devices.

The ruby rod laser was first demonstrated in May 1960 by Ted Maiman of the Hughes Research Laboratory in California. Using a high-intensity flash lamp to excite the electrons in a ruby rod with silvered (mirror) ends, Maiman was able to stimulate the emission of an amplified, high-powered beam of red

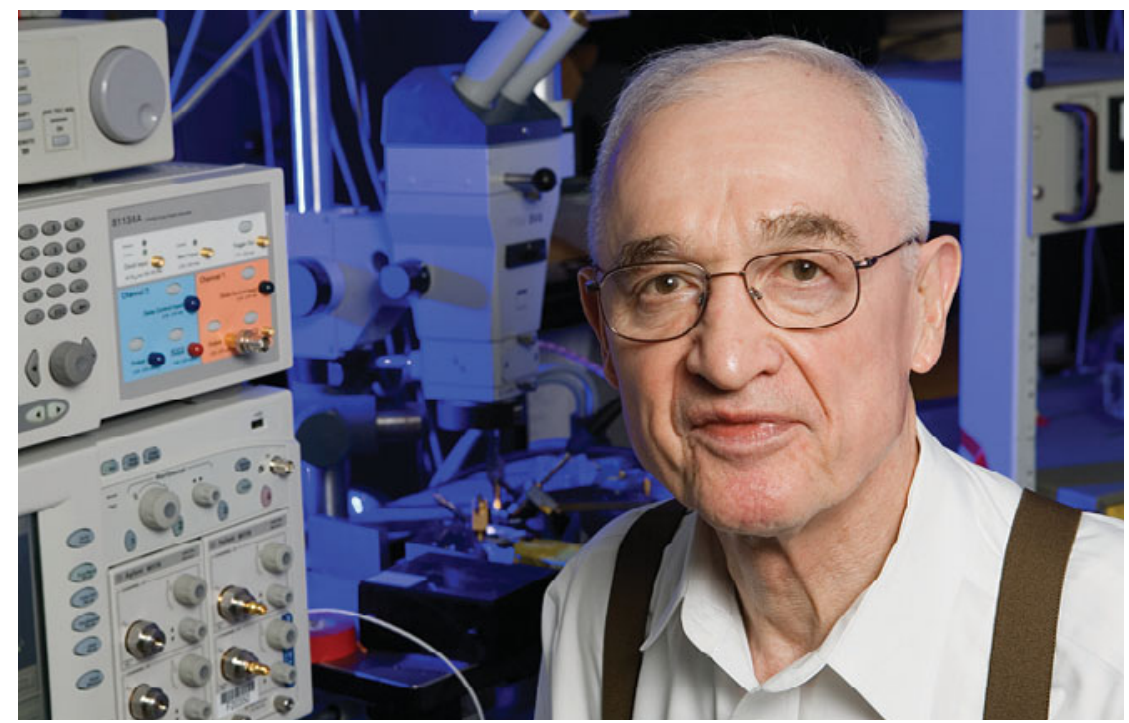

Nick Holonyak Jr., invented the visible light-emitting diode. Photo by L. Brian Stauffer 
light. This beam streamed through the end of the rod that was only partially silvered. The demonstration proved for the first time that light could be made coherent. Prior to this demonstration, the possibility of producing coherent light was hotly debated by scientists. After all, every light source known until then was incoherent. There was even a debate about whether the human eye would be able to see coherent light if it were created, because the eye had evolved in a world in which coherent light did not exist. Maiman had silenced this debate, and generated a high level of excitement in the scientific world. What could laser light be used for, and what other ways could it be generated?

Researchers around the world were soon producing lasers from other solid materials, such as calcium fluoride, and from gases, such as a helium/neon mixture. Not to be outdone, semiconductor scientists entered the fray, competing to see who could invent the first semiconductor diode laser.

In this technological race, some semiconductor researchers took the logical, most direct route to the finish line: They worked with gallium arsenide (GaAs), an off-the-shelf semiconductor material that, if successful, would generate laser light in the infrared. But infrared radiation is invisible. After Holonyak saw a Maiman ruby rod laser in operation at General
Electric, he immediately set a loftier goal for himself. "I wanted visible light," Holonyak said. "I wanted to see what I was doing." This would require perfecting a method he had been working on to create more complex semiconductorsthree-element alloys such as gallium arsenide phosphide (GaAsP) that many scientists believed were impossible to make. But he was determined to follow this harder path. Holonyak said, "I wanted to see from a semiconductor, the kind of light Maiman could see from ruby."

$$
* * * * *
$$

Holonyak began working with semiconductors in 1952 as a graduate student in electrical engineering at the University of Illinois at Urbana-Champaign. Luckily for him, in 1951, John Bardeen, co-inventor of the transistor, had left industry to teach and continue his semiconductor research at the same university. When Bardeen established a semiconductor research laboratory in 1952, Holonyak immediately left the vacuum tube group where he was researching microwave vacuum tubes to become Bardeen's first graduate student. He made the move despite the warnings of some of his colleagues that he was betting on the wrong technology. It was the start of a scientific collaboration and friendship that would end only with Bardeen's death in 1991; Holonyak

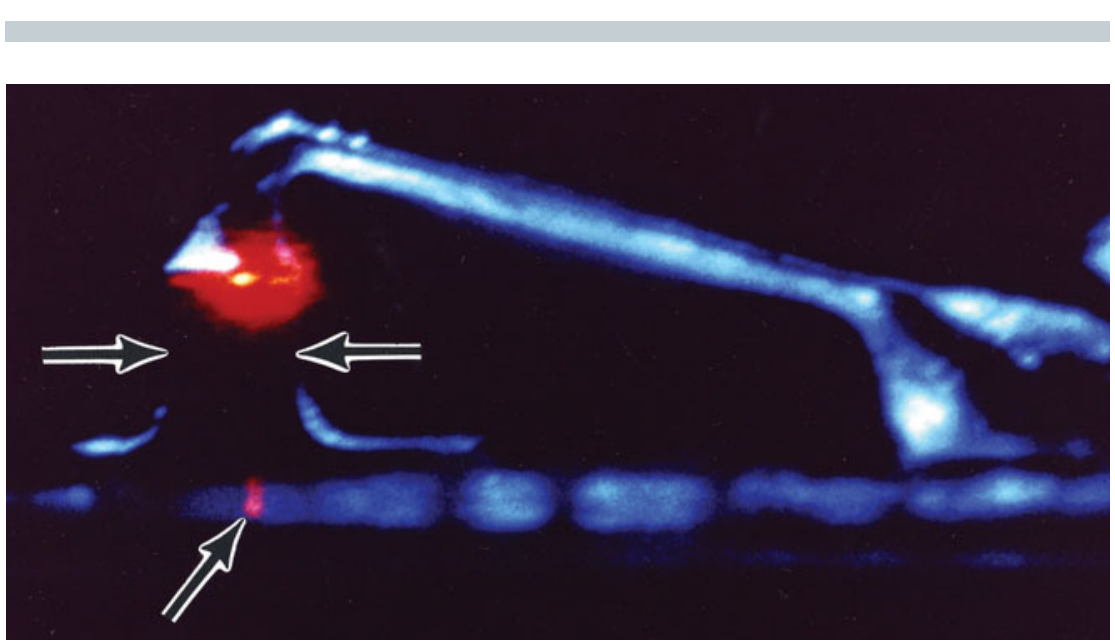

First red-spectrum $\mathrm{GaAs}_{1-x} \mathrm{P}_{x}$ diode laser photographed with its own red laser radiation recorded (red) on film. This is also the basis for the ubiquitous red GaAsP light-emitting diode (LED). Horizontal arrows delineate the diode; the other arrow points out a diffraction spot. Credit: Holonyak, GE, Syracuse, 1962 continues to honor his mentor to this day.

After two years of working with germanium semiconductors under Bardeen's tutelage, Holonyak graduated with his $\mathrm{PhD}$ degree in 1954. He then took a job at Bell Labs in New Jersey for a year, where he had what he calls the "good fortune" of working for research group leader John Moll. Moll was tasked with developing a massive, solid-state switching system that could replace the faulty electromechanical system based on vacuum tubes then in use. The clear favorite semiconducting material for telephone switching devices was silicon (a Group IV element) and Moll wanted Holonyak to devote all his efforts there. But by this time Si technology was relatively sophisticated, at least by Holonyak's standards. "I was restive about some other ideas," he recalls. "I wanted to keep working on stuff that was pioneering. The III-Vs were bothering me."

The main thing that Holonyak wanted to investigate using the III-V compounds was the hypothesis that a simple $p-n$ junction- just a part of the $n-p-n$ transistor-could be used to generate light. The reasoning was as follows: $n$-type materials have an excess of negatively charged, high-energy electrons, while $p$-type materials are dominated by lower energy holes. Holes were originally a theoretical construct meant to represent the absence of an electron in the lattice structure of a solid, but by the 1950 s it became apparent that holes in a solid were real entities. "If you can yank an electron out of the valence band, and kick it up higher in energy, you put the electron up where it can conduct, and down below you've left a void," Holonyak said. "Now that void, that hole, you can't squirt out of the solid into the vacuum, but inside the semiconductor it's as real as the electron is."

So it was thought that if a $p-n$ junction was made part of an electric circuit, with the positive terminal of a battery connected to the $p$-type side and the negative terminal to the $n$-type side, the electrons and holes would be propelled toward the $p-n$ junction. Meeting there, the higher energy electrons would recombine with the lower energy holes, 


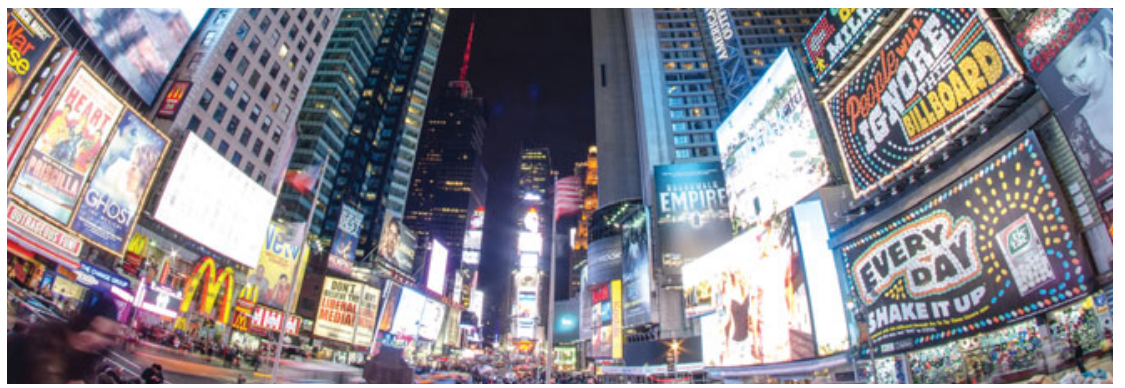

New York City, Times Square LED lights, made possible by Holonyak's invention of the visible light-emitting diode 50 years ago. Photo by Giancarlo Gagliardi/Shutterstock.com

and a photon of light with energy equal to the energy bandgap of the semiconductor would be emitted for each recombination event. The device would thus be a light-emitting diode.

Holonyak spent only one year at Bell Labs before joining the Army Signal Corps in 1955. But during that year at Bell he made significant contributions to the science of diffused-impurity silicon devices, including silicon transistors, oxidemasked transistors, and silicon-controlled rectifiers. Upon leaving the Army in 1957, he joined General Electric in Syracuse, New York, where he invented the fourlayer $p-n-p-n$ thyristor that made the dimmer switch possible. He also worked on tunnel diodes and many other technologies while continuing his research into III-V materials. But when Maiman demonstrated his ruby rod laser in 1960, Holonyak immediately joined the race to invent a semiconductor diode laser.

$$
* * * * *
$$

The first step in the competition was choosing a material. Researchers at Bell Labs and at Phillips in Europe chose gallium phosphide (GaP), which would, if successful, emit red light. But GaP had what was called an "indirect gap" - the momentum of the holes and the electrons did not match, so the connection between them would be weak when it came to recombining at the $p-n$ junction. Holonyak and others decided to focus on "direct gap" materials, in which the electron momentum equaled the hole momentum, making it a more reliable, efficient emitter.
Ultimately it came down to two researchers from General Electric in New York-Robert Hall at the Schenectady lab and Holonyak at the Syracuse lab. Hall was not committed to producing a visible LED, so he used an off-the-shelf III-V crystal of GaAs, a direct-gap material that would emit light in the infrared region of the spectrum. Holonyak had settled on GaAsP as his best bet, despite opposition. "When I was trying to make GaAsP," Holonyak said, "people disputed with me, saying no, no, no, alloys are wrong, alloys are hard to make, they'll be lumpy, they'll be inhomogeneous." Showing his usual optimism, Holonyak recalls, "I was of the opinion that 'I can make this stuff.",

Why go to all this trouble? Holonyak knew that replacing some of the As atoms in the GaAs crystal with lighter P atoms would make the crystal structure contract slightly, so that the energy gap would shift from the infrared energy bandgap of GaAs to the shorter-wavelength visible red region of the spectrum. He could thus achieve his goal of "seeing what he was doing" while maintaining the desired direct-gap properties of GaAs.

To make GaAsP, Holonyak borrowed from the work of J.C. Marinace of IBM, who demonstrated that iodide gas in a heated, closed quartz tube could transport germanium - an otherwise difficult element to transport due to its nonvolatility - to a cooler part of the tube and deposit it on a seed crystal of germanium. Though it wasn't obvious that the same procedure would work for transporting Ga from compounds such as GaAs and $\mathrm{GaP}$, Holonyak investigated the proce-

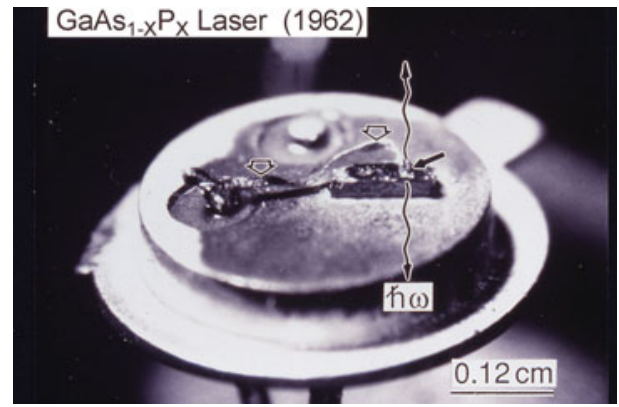

First red-spectrum III-V alloy $\mathrm{GaAs}_{1-x} \mathrm{P}_{x} p-n$ junction laser (October 1962). Reprinted with permission from Nick Holonyak and S.F. Bevacqua, Appl. Phys. Lett. 1 (1962), p. 82. (c) 1962 American Institute of Physics.

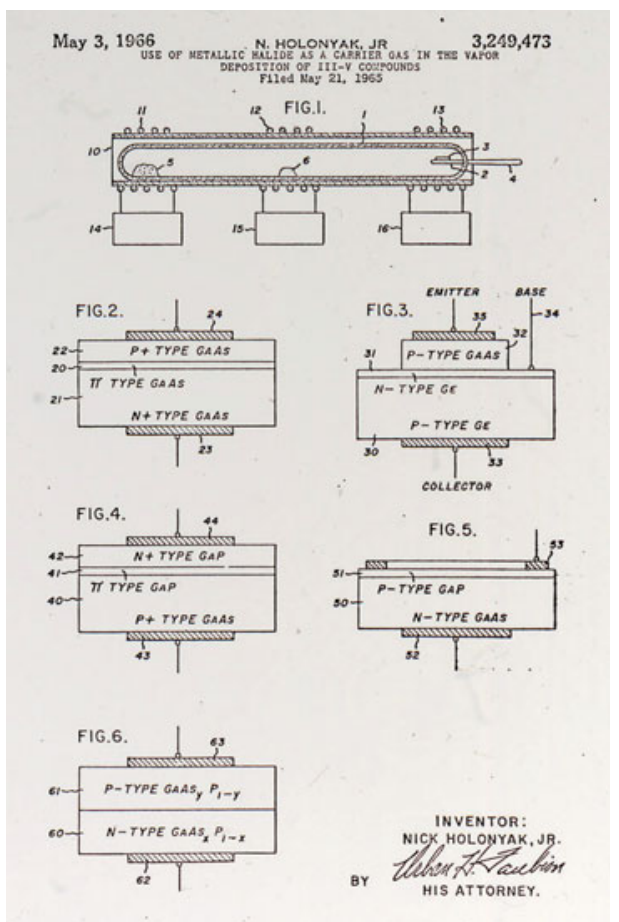

Patent drawing (first filing 1961) of Holonyak's method of closed ampoule vapor-phase epitaxial growth of III-V crystals, including GaAsP and various heterojunctions.

dure in detail in 1959 and 1960. He found that chlorine could be used to transport nonvolatile $\mathrm{Ga}$ in a heated, sealed quartz tube. This made it possible for him to build GaAsP crystals bit by bit.

The procedure he eventually settled on involved placing crystals of GaAs and $\mathrm{GaP}$ in one end of a sealed quartz tube along with chlorine gas. The temperature at this end of the tube was held at $1000-1100^{\circ} \mathrm{C}$, while the other end was at a cooler $850-950^{\circ} \mathrm{C}$. The volatile As 
and $\mathrm{P}$ atoms would easily evaporate from their crystals, while at the same time the chlorine was attacking the Ga and transporting it to the cooler end of the tube. The Ga, As, and $\mathrm{P}$ atoms would then deposit on the cool walls of the other end of the tube simultaneously to form GaAsP crystals with none of the predicted lumpiness or inhomogeneity. By substituting zinc chloride, tin chloride, or other chloride compounds for the chlorine gas, he could dope the crystals to produce $n$-type or $p$-type GaAsP at will, thereby allowing him to create $p-n$ junctions in the material. Holonyak was awarded US Patent number 3,249,473 for this process in 1966.

The only remaining challenge was to make a laser out of the material. This meant creating a laser cavity. A laser cavity is a region in which the light waves bounce back and forth to stimulate additional radiation. Maiman had coated the ends of his ruby rod laser with silver mirrors to create his laser cavity. Both Hall and Holonyak opted for what seemed an easier route - creating FabryPérot facets on the faces of the crystal. Fabry-Pérot facets are perfectly parallel opposite faces of a crystal, like the opposite faces of a cube. These facets act as mirrors, but making them was not easy.

Holonyak attempted to create the parallel faces by cleaving the opposite faces, but this failed because he was not working with single crystals. When he learned that Hall was using the alternative technique of gently polishing the opposing faces of his GaAs crystals to make the parallel facets, he switched to that technique.

But it was too late. In September 1962, Hall reported the successful operation of the first infrared semiconductor laser. Holonyak was disappointed when he heard the news. "But no, Hall beat me," he concedes. "Hall's a great guy, a smart guy, and he got there ahead of me." But Hall's laser LED was not in the visible spectrum.

Holonyak succeeded in demonstrating the red semiconductor laser about three weeks later, on October 9. "Maiman's laser sparkled in the red in 1960 and mine sparkled red in 1962,

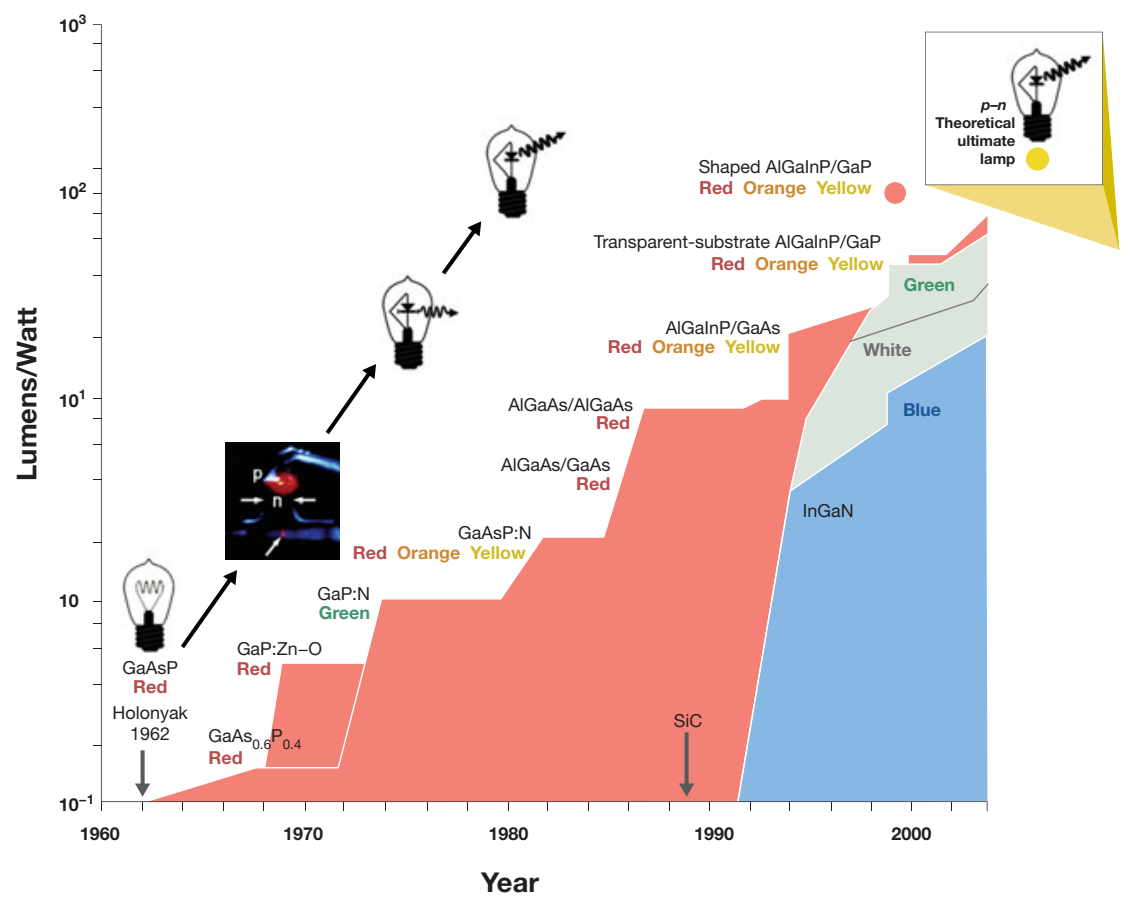

The evolution of the light-emitting diode allong the so-called "Alloy Road."

the first visible semiconductor laser and LED," Holonyak said. Being in the visible range meant that this red laser was also the first semiconducting LED. So despite what looked like a second-place finish, Holonyak was duly credited with being the inventor of the LED.

But beyond that distinction, Holonyak knew that he had developed a technology that held the potential to create LEDs of any color by tuning the energy bandgap of the semiconductor $p-n$ junction. The tuning could be done by changing the elements in the semiconductor crystal to create different alloys with different bandgap energies. This systematic method of creating LEDs spanning the colors of the visible spectrum became known as the "alloy road."

Holonyak pursued the alloy road back at the University of Illinois at UrbanaChampaign after accepting John Bardeen's invitation to join the faculty in 1963. He soon had graduate students under his wing, and the work of creating LEDs and lasers of many colors became the thesis work of some of them. By adding the Group III elements aluminum and indium to the mix, his students ex- panded the range of possible bandgaps, including the InGaP alloy that led to red, orange, yellow, and green LEDs. Holonyak's group was also the first to make quaternary (four-element) alloys such as InGaPAs and AlGaAsP. Under his guidance they invented quantum-well lasers and LEDs and the truncated-invertedpyramid for extracting more light from an LED, among many other innovations.

Now 83, Nick Holonyak holds, appropriately, the John Bardeen Chair in Electrical and Computer Engineering at the university, and is still doing cuttingedge research on optoelectronic devices. In 2004, he and colleague Milton Feng invented the first transistor-laser, combining the amplifying properties of the transistor co-invented by his friend and mentor Bardeen with the coherent-lightemitting ability of Maiman's laser.

But despite his many contributions to LED technology, Holonyak is quick to spread the credit around. "Thousands of people have contributed to it, that's the status of the LED at this point," he said. "There's only a little bit of it that's mine - the front-end stuff." 